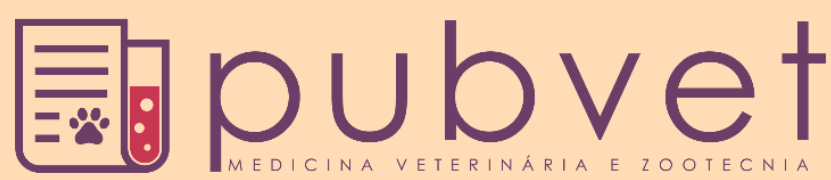

https://doi.org/10.31533/pubvet.v15n01a739.1-6

\title{
Influência da inclusão da raspa de mandioca sobre desempenho, rendimento de carcaça e qualidade da carne de codornas de corte
}

\author{
Roberto Sobral Félix ${ }^{1^{*}} \theta$, Emanuel de Souza Oliveira $^{1} \theta$, Allan Cerqueira de Oliveira ${ }^{1} \theta$, \\ Guilherme Rodrigues do Nascimento ${ }^{2 \Phi}$, Cleidida Barros de Carvalho ${ }^{2 \Phi}$, Clementina Cândida \\ Barros de Carvalho Santos 2
}

${ }^{I}$ Discente do curso de Bacharelado em Zootecnia- Instituto Federal de Educação, Ciências e Tecnologia Baiano, Campus Santa Inês - Bahia, Brasil. ${ }^{2}$ Professor(a) no Instituto Federal de Educação, Ciências e Tecnologia Baiano, Campus Santa Inês - Bahia, Brasil.

*Autor para correspondência, E-mail: robertosobralfelix@gmail.com

Resumo. O presente estudo foi conduzido com o objetivo de avaliar o desempenho, rendimento de carcaça e qualidade da carne de codornas alimentadas com diferentes níveis $(0,6,12$ e $18 \%)$ de inclusão da raspa de mandioca na dieta de codornas. Foram utilizadas 100 codornas de corte nativas da região, em um lote misto de sexo, onde foram avaliados os parâmetros de rendimento de carcaça, rendimento de cortes nobres (peito e coxa mais sobrecoxa), rendimento da moela, $\mathrm{pH}$. Atividade água ( $\mathrm{Aw}$ ) e luminosidade ( $\left.\mathrm{L}^{*}, \mathrm{a}^{*}, \mathrm{~b}^{*}\right)$. Não foi observado diferença entre os tratamentos das variáveis analisadas, sendo possível aumentar o nível da raspa de mandioca na alimentação de codornas, ficando necessária a adição de pigmentantes na ração, visto que ela diminui a incidência de vermelho ( $\mathrm{a}^{*}$ ) na carne.

Palavras-chave: Cor, qualidade, ração

\section{Influence of cassava zest inclusion on performance, carcass yield, and meat quality of beef quails}

\begin{abstract}
The present study was conducted to evaluate carcass yield and meat quality of quails fed different levels of cassava zest in the quail diet. A total of 100 native quails from the region were used in a mixed-sex group, where the parameters of carcass yield, noble cuts (breast and thigh plus thigh), gizzard yield, $\mathrm{pH}$ were evaluated. Water activity (Aw) and luminosity $(\mathrm{L} *, \mathrm{a} *, \mathrm{~b} *)$. No difference was observed between the parameters of the analyzed variables, and it was possible to increase the level of cassava zest in the quail feed, allowing the addition of pigments in the ratio since it decreased the incidence of red $(\mathrm{a} *)$ in meat.
\end{abstract}

Keywords: Feed, color, quality

\section{Introdução}

A coturnicultura leva uma série de vantagens em relação as criações de outras aves, são elas: a precocidade sexual, o ganho de peso rápido, a resistência a parasitas que geralmente atacam as outras aves, resistência ao calor, e maior número de animais por metro quadrado (Mendez, 2015).

Em 2016 o Brasil sofreu uma redução de $20 \%$ no efetivo de codornas e essa diminuição de produção afetou as cadeias produtivas de ovos e carne, a razão pelo ocorrido é justificada pelo declínio na renda dos consumidores (IBGE, 2017).

Um fator que afeta de forma negativa a pecuária é a sazonalidade das culturas que provoca em determinada época do ano a escassez de alimento. A falta de tecnologias para enfrentar tais problemas, 
ocasiona muitos danos na produção animal, contudo a coturnicultura se torna uma maneira viável para driblar esse contratempo, visto que as codornas por serem animais menores, tendem a consumir menos ração em relação às outras aves de produção.

As rações têm um papel fundamental, tanto no desempenho das aves quanto no custo de produção, onde essas despesas podem chegar a 75\% do mesmo (Murakami \& Ariki, 1998). Com isso, alguns produtores rurais vêm buscando alimentos alternativos, a fim de diminuir esses custos com as rações; no semiárido nordestino a mandioca vem sendo muito utilizada como subproduto na alimentação animal.

A mandioca é bastante difundida no Brasil e a mesma fica entre os primeiros colocados na posição mundial de produção, $80 \%$ da produção nacional é utilizado para fazer farinha, principalmente na região nordeste. $\mathrm{O}$ subproduto como a raspa e parte aérea é utilizada para a alimentação animal (Caldas Neto et al., 2000).

Em frangos de corte caipira a substituição do milho pela raspa de mandioca não teve uma diferença significativa no ganho de peso, entretanto, a coloração de pele se tornou mais clara, o que não é um atrativo no mercado de frangos caipira (Carrijo et al., 2010).

O presente estudo objetivou avaliar o desempenho, rendimento de carcaça e qualidade da carne de codornas alimentadas com diferentes níveis de inclusão da raspa de mandioca na dieta.

\section{Material e métodos}

O experimento foi conduzido no setor de coturnicultura pertencente ao Instituto Federal Baiano, localizado no município de Santa Inês, que compete a mesorregião do centro-sul baiano e o semiárido nordestino, e as análises de qualidade foram realizadas no laboratório de carnes do próprio instituto.

Foram utilizadas 100 codornas para corte, em um lote de sexo misto, alojados em gaiolas, de arame galvanizado com o comprimento: $31 \mathrm{~cm}$; Largura: $25 \mathrm{~cm}$; Altura: $25 \mathrm{~cm}$, alimentadas com ração e água à vontade durante toda a fase de criação. Durante os 10 primeiros dias as aves foram criadas com ração comercial inicial para codornas, posteriormente foram distribuídas nas gaiolas e fornecidas as rações experimentais.

As codornas foram distribuídas em gaiolas experimentais, com delineamento inteiramente casualizado (DIC), sendo quatro tratamentos $(\mathrm{T} 1=0 \%, \mathrm{~T} 2=6 \%, \mathrm{~T} 3=12 \%$ e $\mathrm{T} 4=18 \%$ de inclusão de raspa de mandioca) e cinco repetições. Foi formulada apenas uma ração para todo o período experimental 10 a 35 dias, com base nas exigências nutricionais das codornas, segundo Silva \& Costa (2009).

A raspa da mandioca é oriunda do processo de fabricação da farinha, então o subproduto utilizado no experimento, foi doado por agricultores da região. Para triturar a raspa, é necessário de uma umidade baixa, o que foi conseguido com secagem por exposição ao sol durante quatro dias, com a intenção de facilitar a moagem e evitar a proliferação de fungos.

Aos 35 dias de idade, foram retiradas duas aves de cada unidade experimental, de acordo com o peso médio $( \pm 5 \%)$ da parcela. As aves foram identificadas, insensibilizadas por deslocamento cervical, abatidas e sangradas por dois minutos, após jejum de sólidos de seis horas. A escaldagem das aves foi realizada a uma temperatura controlada de 53 a $55^{\circ} \mathrm{C}$, por 20 a 40 segundos. Logo após, as aves foram evisceradas, retiradas os pés, cabeça e levada para análises de qualidade. Dentro dos parâmetros avaliados, estão: rendimento de carcaça, rendimento de cortes nobres (peito e coxa mais sobrecoxa), rendimento da moela, $\mathrm{pH}$. Atividade água (Aw) e luminosidade $\left(\mathrm{L}^{*}, \mathrm{a}^{*}, \mathrm{~b}^{*}\right)$.

A luminosidade foi determinada pelo uso do colorímetro (Minolta CR400) com abertura de $8 \mathrm{~mm}$ de diâmetro, no sistema CIELAB, sendo avaliados os parâmetros L* (luminosidade), a* (+a indica vermelho e $-a$ indica verde) e $b^{*}$ (+b indica amarelo e $-b$ indica azul). Os valores $L^{*}, a^{*}$ e $b^{*}$ foram medidos em três diferentes pontos na superfície ventral e no meio da seção cranial do músculo Pectoralis major.

Para as leituras de $\mathrm{pH}$ (média de três leituras), utilizou-se um eletrodo de penetração (Simpla PH140), diretamente no músculo Pectoralis major. 
Utilizou o analisador de atividade de agua (Aw) NOV-LABSWIFT para os dados de Aw, que tem uma faixa de medição de 0,0300 a 1,000 Aw (3 a 100\% Umidade Relativa), com resolução de 0,001 Aw $+/-0.1^{\circ} \mathrm{C}$; precisão: $+/-0,003 \mathrm{Aw}\left(+/-0,1^{\circ} \mathrm{C}\right)$. Foi retirada duas amostras do Pectoralis major de cada repetição e obtida a média. As rações experimentais foram formuladas com o auxílio do super Crac 5.7 versão máster.

Tabela 1. Ração de 10 a 45 dias para codornas com níveis distintos de Raspa de Mandioca.

\begin{tabular}{|c|c|c|c|c|}
\hline \multirow{2}{*}{ Ingredientes } & \multicolumn{4}{|c|}{ Níveis de Inclusão \% } \\
\hline & 0 & 6 & 12 & 18 \\
\hline Milho grão & 54,44 & 48,18 & 41,93 & 35,67 \\
\hline Soja farelo $45 \%$ & 39,08 & 39,08 & 39,08 & 39,08 \\
\hline Mandioca int. Raspa & 0,00 & 6,00 & 12,00 & 18,00 \\
\hline Óleo de soja & 2,58 & 2,84 & 3,10 & 3,36 \\
\hline Premix-app & 1,50 & 1,50 & 1,50 & 1,50 \\
\hline Fosfato bicálcico & 0,92 & 0,93 & 0,95 & 0,96 \\
\hline Calcário & 1,07 & 1,05 & 1,03 & 1,01 \\
\hline Sal comum & 0,35 & 0,35 & 0,35 & 0,34 \\
\hline Lisina & 0,04 & 0,06 & 0,07 & 0,09 \\
\hline Total (kg) & 100,00 & 100,00 & 100,00 & 100,00 \\
\hline \multicolumn{5}{|l|}{ Composição nutricional } \\
\hline Proteína Bruta & 22 & 22 & 22 & 22 \\
\hline Energia Metabolizável (Kcal/kg) & 2.950 & 2.950 & 2.950 & 2.950 \\
\hline Cálcio & 0,75 & 0,75 & 0,75 & 0,75 \\
\hline Fósforo disponível & 0,29 & 0,29 & 0,29 & 0,29 \\
\hline Sódio & 0,16 & 0,16 & 0,16 & 0,16 \\
\hline Lisina digestível & 1,14 & 1,14 & 1,14 & 1,14 \\
\hline
\end{tabular}

Os dados coletados foram submetidos à análise de variância e ao teste de comparação de médias de Dunnet a 5\% de probabilidade. Quando significativo $(\mathrm{P}>0,05)$, foi realizado análise de regressão, possibilitando determinar o melhor nível de inclusão da raspa de mandioca a ser estimado por meio de equações de regressão, respeitando-se a interpretação biológica das variáveis.

\section{Resultados e discussão}

Nos parâmetros de consumo de ração $(\mathrm{g})$ e ganho de peso $(\mathrm{g})$, observou efeito positivo $(\mathrm{P}>0,05)$ na inclusão de $18 \%$ de raspa de mandioca em relação ao tratamento controle. Para a conversão alimentar não teve interferência $(\mathrm{P}>0,05)$ sobre os tratamentos.

Não foi observado os mesmos resultados quando Geron et al. (2015) avaliaram o desempenho de frangos de cortes alimentados com a raspa de mandioca, onde, o mesmo encontrou uma linearidade negativa com o aumento do nível de inclusão desse subproduto para os parâmetros de ganho de peso e conversão alimentar. A não similaridade nos experimentos deve estar relacionada aos méritos genéticos das aves, visto que as codornas tendem a ser um animal mais rústico e com facilidade para adaptação as mais diversas fontes de alimentação. Em estudos com aves, Holanda et al. (2015), utilizaram 5 níveis distintos de raspa de mandioca, $(0 \%, 12,24,36$ e $48 \%)$ e constataram que pode ser substituído até o último nível, contudo, pode-se testar níveis mais altos de inclusão da raspa de mandioca para avaliações dos parâmetros da Tabela 2.

Tabela 2. Desempenho de codornas (Coturnix coturnix) de corte alimentadas com ração contendo raspa integral de mandioca.

\begin{tabular}{lcccccc}
\hline \multirow{2}{*}{ Característica } & \multicolumn{4}{c}{ Raspa de mandioca, \% } & P-Valor \\
\cline { 2 - 5 } & 0 & 6 & 12 & 18 & \\
\hline Consumo de ração $(\mathrm{g})$ & $228,6 \pm 13$ & $255,5+12,5$ & $266,5 \pm 17,6$ & $317,9^{*} \pm 27,1$ & 0,035 \\
Ganho de peso(g) & $76,92 \pm 7,82$ & $92,25 \pm 6,5$ & $79,5 \pm 8$ & $104^{*} \pm 2,32$ & 0,036 \\
Conversão Alimentar & $3,204 \pm 0,267$ & $2,803 \pm 0,159$ & $3,471 \pm 0,342$ & $3,068 \pm 0,281$ & 0,382 & 18,67 \\
\hline
\end{tabular}

*Difere do controle pelo teste de Dunnett $(\mathrm{P}<0,05)$.

Corroborando com os resultados, Farias et al. (2014), acharam valores semelhantes de conversão alimentar de codornas quando avaliou a inclusão do farelo de arroz integral em substituição parcial do milho, segundo ele até o nível de $20 \%$ não interfere na produção de codornas de corte. 
Na Tabela 3 são apresentadas as comparações de medias $(P \geq 0,05)$, e o coeficiente de variação para os dados de luminosidade $\left(\mathrm{L}^{*}\right)$, intensidade de vermelho $\left(\mathrm{a}^{*}\right)$ intensidade de amarelo $\left(\mathrm{b}^{*}\right)$. As médias seguidas de $\left(^{*}\right)$ apresentam efeito em relação ao tratamento controle.

Tabela 3. Análise colorimétrica, $\mathrm{pH}$ e atividade de água em peito de codornas (Pectoralis major) alimentadas com raspa de mandioca.

\begin{tabular}{|c|c|c|c|c|c|c|}
\hline \multirow{2}{*}{ Característica } & \multicolumn{4}{|c|}{ Raspa de mandioca, $\%$} & \multirow{2}{*}{ P-Valor } & \multirow{2}{*}{$\mathrm{CV}$} \\
\hline & 0 & 6 & 12 & 18 & & \\
\hline $\mathrm{L}^{*}$ & $56,12 \pm 1,39$ & $58,39 \pm 1,87$ & $53,08 \pm 1,49$ & $55,44 \pm 1,34$ & 0,127 & 9,05 \\
\hline$a^{*}$ & $3,48 \pm 0,321$ & $3,77 \pm 0,49$ & $3,41 \pm 0,35$ & $2,68 \pm 0,279^{*}$ & 0,045 & 35,87 \\
\hline$b^{*}$ & $11,04 \pm 0,70$ & $12,12 \pm 1,00$ & $9,86 \pm 0,77$ & $10,43 \pm 0,37$ & 0,188 & 22,23 \\
\hline $\mathrm{pH}$ & $5,2470 \pm 0,0687$ & $5,2930 \pm 0,0902$ & $5,1380 \pm 0,0728$ & $5,0100 \pm 0,0269$ & 0,029 & 4,57 \\
\hline Aw & $0,885 \pm 0,005$ & $0,886 \pm 0,010$ & $0,901 \pm 0,009$ & $0,903 \pm 0,005$ & 0,235 & 2,83 \\
\hline
\end{tabular}

*Difere do controle pelo teste de Dunnett $(\mathrm{P}<0,05)$.

Segundo Pinheiro et al. (2015), a coloração da carne influencia no mercado consumidor, sendo de extrema importância a mantença das características e coloração da carne de codorna para uma boa aceitação no mercado.

Nenhum dos dados de $\mathrm{L}^{*}$ e $\mathrm{b}^{*}$ obtiveram diferença significativa $(\mathrm{P}>0,05)$, portanto a dieta com maior inclusão da raspa de mandioca não interfere para essas variáveis (Tabela 3 ).

Em estudos relacionados a qualidade de carne de codornas, Sanfelice et al. (2010), encontrou uma média de $\mathrm{L}^{*}$ de 52,2; segundo o autor esse valor se torna ruim para o mercado consumidor, visto que a carne tem uma tendência maior para a palidez. Levando em consideração essa afirmação, os resultados encontrados não foram satisfatórios para a comercialização, precisando então, de pigmentantes para elevar a luminosidade.

Corroborando com os resultados, Lara et al. (2002) dizem que o $\mathrm{pH}$ abaixo de 5,2 e o valor de $\mathrm{L}^{*}$ acima de 52 aferidos após as 24 horas, caracteriza um início de uma carne PSE em frangos, contudo, os valores de $\mathrm{L}$ encontrado no experimento foram superior a 52,0; isso se dá por conta das codornas terem uma característica de carne mais escura. O pH também fica na faixa de 5,1 a 5,3, dando uma característica da carne de codorna ser PSE.

De acordo com Abreu et al. (2014), para a carne de codornas se enquadrar na normalidade, deve estar com o pH por volta de 5,7. Valores semelhantes foram encontrados por Pinheiro et al. (2015), quando avaliou diferentes níveis de proteínas na alimentação de codornas.

As medias de atividade de água (Aw), girou entorno de 0,9 e não tiveram efeito em relação ao controle. De acordo com Cunha (2020), o desenvolvimento microbiano em alimentos com Aw entre 0,7 e 1 é muito alto. Com isso, o resfriamento e congelamento são elementos imprescindíveis para uma durabilidade no armazenamento dessas carcaças, visto que eles diminuem e cessam esse fator respectivamente.

O teor de mioglobina é o que determina a coloração da carne, quanto maior o teor dessa proteína, a carne aumenta a sua coloração com tendência para o vermelho. Esse fator também é influenciado pelo movimento, quanto maior for à atividade física do animal, maior é o teor de mioglobina no musculo.

Houve diferença significativa para $\mathrm{a} *(+\mathrm{a}$ indica vermelho e $-\mathrm{a}$ indica verde) nos tratamentos 1 e 4 que se refere a inclusão de 0 e $18 \%$ de raspa. Os valores encontrado para a* se aproximaram com as medias encontradas por Santos et al. (2005), quando ele avaliou a coloração de frangos caipiras. A similaridade de cor entre codornas e frangos caipiras se deve pelas características de movimentos parecidos, visto que os dois são animais bastante agitados.

Para valores de $b^{*}$ foram observados predominância da tonalidade amarela, o que pode estar relacionado com a presença de carotenoides no milho, principalmente o zeaxantina, que quando depositado na carne, é responsável pela tonalidade amarela.

Ao se analisar a regressão de $\mathrm{a}^{*}$, teor de vermelho para verde, constatou-se que ao aumentar a inclusão de rapa de mandioca até o nível de 5,77\% não interfere na coloração, sendo esse o ponto de máxima, contudo, a partir desse nível o teor de vermelho é suprimido. A raspa de mandioca teve um 
comportamento semelhante quando Pereira et al. (2016) analisaram o peso do ovo em codornas poedeira, constatando-se que a medida que se aumentava a inclusão de raspa diminui o peso do ovo.

Na Figura 1, o gráfico representa graficamente à regressão linear da variável $\mathrm{a}^{*}$, cuja fórmula matemática é $y=-0,0071 x^{2}+0,082 x+3,494$ e o $R^{2}=0,994$.

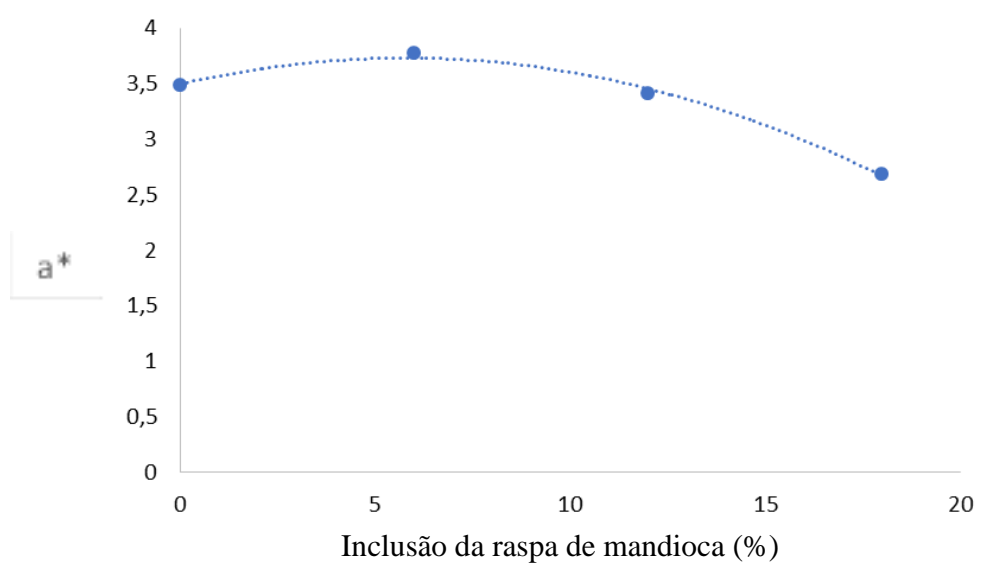

Figura 1. Análise de regressão para a variável a*.

Os cortes nobres e seus rendimentos, como peito, cocha mais sobrecoxa e órgãos internos como moela, não apresentaram diferença, isso se deu por conta da não variação do peso vivo, onde os membros e órgãos internos devem ser proporcionais ao corpo do animal, portanto os dados seguem uma coerência em relação a simetria corporal (Tabela 4).

Tabela 4. Rendimentos de carcaça e cortes de codornas alimentadas com diferentes níveis de Raspa de Mandioca.

\begin{tabular}{|c|c|c|c|c|c|c|}
\hline \multirow{2}{*}{ Características } & \multicolumn{4}{|c|}{ Raspa de mandioca, $\%$} & \multirow{2}{*}{ P-Valor } & \multirow{2}{*}{$\mathrm{CV}$} \\
\hline & 0 & 6 & 12 & 18 & & \\
\hline$\overline{\text { Peso vivo, } \mathrm{g}}$ & $109,50 \pm 6,77$ & $120,00 \pm 6,99$ & $122,50 \pm 7,20$ & $126,00 \pm 7,88$ & 0,418 & 16,15 \\
\hline Carcaça, g & $76,00 \pm 5,42$ & $85,50 \pm 5,50$ & $86,00 \pm 5,57$ & $89,00 \pm 6,62$ & 0,429 & 21,74 \\
\hline Peito, $g$ & $30,38 \pm 1,85$ & $35,33 \pm 2,24$ & $35,14 \pm 1,90$ & $36,91 \pm 2,87$ & 0,214 & 21,13 \\
\hline C e sobrecoxa, $g$ & $21,38 \pm 1,40$ & $23,36 \pm 1,16$ & $24,01 \pm 1,49$ & $23,21 \pm 1,50$ & 0,586 & 18,93 \\
\hline Moela, g & $7,58 \pm 0,32$ & $7,98 \pm 0,22$ & $7,57 \pm 0,41$ & $7,99 \pm 0,25$ & 0,584 & 8,52 \\
\hline \multicolumn{7}{|c|}{ Rendimento } \\
\hline Carcaça \% & $69 \pm 1,071$ & $71,037 \pm 0,724$ & $70,01 \pm 1,04$ & $70,11 \pm 1,17^{\mathrm{a}}$ & 0,704 & 5,36 \\
\hline Peito $\%$ & $40,416 \pm 0,997$ & $41,367 \pm 0,441$ & $41,37 \pm 1,35$ & $41,392 \pm 0,769$ & 0,856 & 7,08 \\
\hline C e sobrecoxa $\%$ & $28,337 \pm 0,552$ & $27,619 \pm 0,650$ & $28,002 \pm 0,595$ & $26,393 \pm 0,756$ & 0,176 & 7,58 \\
\hline Moela \% & $7,127 \pm 0,589$ & $6,542 \pm 0,342$ & $7,357 \pm 0,443$ & $6,893 \pm 0,472$ & 0,695 & 15,66 \\
\hline
\end{tabular}

Avaliando o nível de proteína bruta na dieta de codornas, Pinheiro et al. (2015) não encontrou diferença significativa $(\mathrm{P}>0,05)$ o peso vivo de codornas europeias, chegando a um peso médio de 153 gramas aos 42 dias de vida. Entretanto, o PV encontrado nesse estudo é menor, isso leva a hipótese que pode estar relacionado com os fatores genéticos, condições ambientais e da própria dieta.

Segundo Nascimento et al. (2005), os níveis de inclusão da raspa de mandioca não se deve ultrapassar $10,29 \%$, pois com isso as aves não tem um bom rendimento e esse fator se agrava na fase final da produção, onde os ganhos são mais evidentes.

\section{Conclusão}

A utilização de raspa de mandioca na alimentação de codornas não altera o rendimento de carcaça, o desempenho e rendimento de cortes, entretanto, altera a cor da carne, sendo necessária a adição de pigmentantes na ração no nível de $18 \%$ de inclusão de raspa de mandioca.

\section{Referências}

Abreu, L. R. A., Boari, C. A., Pires, A. V., Pinheiro, S. R. F., Oliveira, R. G., Oliveira, K. M., Gonçalves, F. M., \& Oliveira, F. R. (2014). Influência do sexo e idade de abate sobre rendimento de carcaça e qualidade da carne de 
codornas de corte. Revista Brasileira de Saúde e Produção Animal, 15(1), 131-140. https://doi.org/10.1590/S1519-99402014000100020

Caldas Neto, S. F., Zeoula, L. M., Branco, A. F., Prado, I. N., Santos, G. T., Fregadolli, F. L., Kassies, M. P., \& Dalponte, A. O. (2000). Mandioca e resíduos das farinheiras na alimentação de ruminantes: digestibilidade total e parcial. Revista Brasileira de Zootecnia, 29(6), 2099-2108. https://doi.org/10.1590/S151635982002000600030.

Carrijo, A. S., Fascina, V. B., Souza, K. M. R., Ribeiro, S. S., Allaman, I. B., Garcia, A. M. L., \& Higa, J. A. (2010). Níveis de farelo da raiz integral de mandioca em dietas para fêmeas de frangos caipiras. Revista Brasileira de Saúde e Produção Animal, 11(1), 131-139.

Cunha, H. V. F. (2020). A diferença entre atividade de água (Aw) e o teor de umidade nos alimentos. Acesso.

Farias, N. N. P., Freitas, E. R., Xavier, R. P. S., Braz, N. M., Tavares, T. C. L., Figueiredo, C. W. S., Fernandes, D. R., \& Nascimento, G. A. J. (2014). Farelo integral de arroz parboilizado submetido a armazenamento prolongado para alimentação de codornas de corte. Pesquisa Agropecuária Brasileira, 49(6), 407-415. https://doi.org/10.1590/S0100-204X2014000600001

Geron, L. J. V., Costa, F. G., Moraes, K. J. S., Oliveira, E. M., Gomes, R. D., Pereira, S. R., Silva, A. P., Cruz, C., \& Pelicia, K. (2015). Consumo, desempenho e rendimento de carcaça de frangos de corte alimentados com rações contendo raspa de mandioca residual desidratada. Boletim de Indústria Animal, 72(4), 304-310. https://doi.org/10.17523/bia.v72n4p304

Holanda, M. A. C., Holanda, M. C. R., Vigoderes, R. B., \& Dutra Junior, W. M. (2015). Desempenho de frangos caipiras alimentados com farelo integral de mandioca. Revista Brasileira de Saúde e Produção Animal, 16(1), 106-117. https://doi.org/10.1590/S1519-99402015000100012

IBGE. (2017). Produção da Pecuária Municipal - PPM. Instituto Brasileiro de Geografia e Estatística. https://www.ibge.gov.br/estatisticas-

novoportal/economicas/agriculturaepecuaria/9107producaodapecuariamunicipal.html?\&t=resultados

Lara, J. A. F., Ninov, K., Bonassi, C. A., Ledur, M. C., Nepomuceno, A. L., \& Shimokomaki, M. (2002). Estresse térmico e incidência de carne PSE em frangos. Revista Brasileira de Ciência Avícola, 4(1), 15.

Mendez,M.F. B. (2015). Respostas morfofisiológicas de fêmeas de codornas japonesas (Coturnix coturnix japonica) criadas em diferentes fontes luminosas. Lavras: UFLA.

Murakami, A. E., \& Ariki, J. (1998). Produção de codornas japonesas. Jaboticabal: Funep, 507.

Nascimento, G. A. J., Costa, F. G. P., Amarante Junior, V. S., \& Barros, L. R. (2005). Efeitos da substituição do milho pela raspa de mandioca na alimentação de frangos de corte, durante as fases de engorda e final. Ciência e Agrotecnologia, 29(1), 200-207.

Pereira, A. A., Ferreira, D. A., Griep Júnior, D. N., Lima, C. B., Moura, A. S., \& Lima Júnior, D. M. (2016). Raspa da mandioca para codornas em postura. Acta Veterinaria Brasilica, 10(2), 123-129.

Pinheiro, S. R. F., Dumont, M. A., Pires, A. V., Boari, C. A., Miranda, J. A., Oliveira, R. G., \& Ferreira, C. B. (2015). Rendimento de carcaça e qualidade da carne de codornas de corte alimentadas com rações de diferentes níveis de proteína e suplementadas com aminoácidos essenciais. Ciência Rural, 45(2), 292-297. https://doi.org/10.1590/0103-8478cr20120327

Sanfelice, C., Mendes, A. A., Komiyama, C. M., Cañizares, M. C., Rodrigues, L., Cañizares, G. I., Roça, R. O., Almeida, I. C. L. P., Balog, A., \& Milbradt, E. L. (2010). Avaliação e caracterização da qualidade da carne de peito (Pectoralis major) de matrizes pesadas em final de ciclo produtivo. Food Science and Technology, 30, 166-170.

Santos, A. L., Sakomura, N. K., Freitas, E. R., Fortes, C. M. L. S., Carrilho, E. N. V. M., \& Fernandes, J. B. K. (2005). Estudo do crescimento, desempenho, rendimento de carcaça e qualidade de carne de três linhagens de frango de corte. Revista Brasileira de Zootecnia, 34(5), 1589-1598. https://doi.org/http://dx.doi.org/10.1590/S151635982005000500020.

Silva, J. H. V, \& Costa, F. G. P. (2009). Tabela para codornas japonesas e europeias. Jaboticabal, SP: Funep, 110.

Histórico do artigo:

Recebido: 26 de agosto de 2020.

Aprovado: 14 de outubro de 2020.

Disponível online: 31 de novembro de 2020.
Licenciamento: Este artigo é publicado na modalidade Acesso Aberto sob a licença Creative Commons Atribuição 4.0 (CC-BY 4.0), a qual permite uso irrestrito, distribuição, reprodução em qualquer meio, desde que $o$ autor e a fonte sejam devidamente creditados. 